\title{
Probing hole spin transport of disorder quantum dots via Pauli spin-blockade in standard silicon transistors
}

\author{
Joseph Hillier ${ }^{1}$, Keiji Ono ${ }^{2}$, Kouta Ibukuro ${ }^{1}$, Fayong Liu ${ }^{1}$, \\ Zuo Li ${ }^{1}$, Muhammad Husain Khaled ${ }^{1}$, Harvey Nicholas \\ Rutt $^{1}$, Isao Tomita ${ }^{3}$, Yoshishige Tsuchiya ${ }^{1}$, Koji Ishibashi ${ }^{2}$ \\ and Shinichi Saito ${ }^{1}$ \\ ${ }^{1}$ Department of Electronics and Computer Science, University of Southampton, \\ SO17 1BJ, United Kingdom \\ 2 Advanced Device Laboratory, RIKEN Institute, 2-1 Hirosawa, Wako, Saitama, \\ 351-0198, Japan \\ 3 Department of Electrical and Computer Engineering, National Institute of \\ Technology, Gifu college, 2236-2 Kamimakuwa, Motosu, Gifu, 501-0495, Japan \\ E-mail: J.W.Hillier@soton.ac.uk
}

\begin{abstract}
Single hole transport and spin detection is achievable in standard p-type silicon transistors owing to the strong orbital quantization of disorder based quantum dots. Through the use of the well acting as a pseudo-gate, we discover the formation of a double-quantum dot system exhibiting Pauli spinblockade and investigate the magnetic field dependence of the leakage current. This enables attributes that are key to hole spin state control to be determined, where we calculate a tunnel coupling $t_{c}$ of $57 \mu \mathrm{eV}$ and a short spin-orbit length $l_{S O}$ of $250 \mathrm{~nm}$. The demonstrated strong spin-orbit interaction at the interface when using disorder based quantum dots supports electric-field mediated control. These results provide further motivation that a readily scalable platform such as industry standard silicon technology can be used to investigate interactions which are useful for quantum information processing.
\end{abstract}




\section{Introduction}

Silicon ( $\mathrm{Si}$ ) based devices have become the cornerstone of modern technology, and are now a leading candidate for quantum information processing archi- ${ }_{86}$ tectures [1, 2, 3, 4. Promising alternatives to traditional metal-oxide-semiconductor field-effect transistors (MOSFETs) take the form of quantum dots (QDs) as qubits in single electron devices through the use of ${ }_{90}$ charge and spin as the fundamental building blocks $\mathrm{s}_{91}$ [5. 6, 7. The use of multi-gate device architectures ${ }_{92}$ in particular has led to many breakthroughs by tuning the potential profiles defining QDs as well as the inter-dot tunneling barriers for precise control, where $e_{95}$ development is undertaken by academia and large pre- ${ }_{96}$ industrial fabrication facilities $[8$, 9, 10, 11, 12, 13, 14. 14.97 Using Si for spin based quantum computing regimes ${ }_{98}$ is a natural choice for not only scalability and ease of integration with industrial fabrication techniques, but also long coherence times owing to isotopically enriched ${ }_{01}$ zero nuclear spin ${ }^{28} \mathrm{Si}$ [15, 16. The feasibility of spin ${ }_{02}$ qubits has also been demonstrated by high fidelitys exceeding $99.8 \%$, which can be utilized in combination with quantum error correction towards achieving fault tolerant quantum computing, promoting spin as a competitive candidate in this space with respect to trapped ion and superconducting platforms [17, 18, 19, 20, 21.

Efforts have also been made to take advantage of features already present at the single electron level in Si VLSI technology. The use of trap states within Si quantum devices can complement and even enhance single charge transport, with applications ranging from quantum information and quantum metrology, to bio-sensing and hardware security [22, [23, 24, 25]. In quantum metrology, record high frequency benchmarking for single electron pump operation was achieved as a consequence of a coupling between a QD and trap state, yielding improved operation for establishing a new current standard [26, 27]. In addition, hole spin resonance of trap states within a ptype MOSFET was achieved using Pauli spin-blockade (PSB) to study spin-orbit (SO) state mixing 28 .

Alongside scalability, another technological obstacle to overcome is that of electric-field mediated control, which relies on a strong SO interaction, but in the case of electrons in Si is intrinsically weak [29, 30, 31. This has given rise to investigations into transport schemes with an enhanced SO interaction, where attempts using holes, valley states and the inversion asymmetry at the oxide interface has led to strong $\mathrm{SO}^{04}$ effects [32, 33, 34, 35, 36. PSB, a common measure ${ }^{105}$ ment tool used throughout quantum information pro- ${ }^{106}$ tocols, is also dependant on the SO interaction when ${ }^{107}$ detecting spin states [37, 38, 39, 40, 41, 42, 43. This is manifested as the suppression of current through $\mathrm{a}^{109}$ double-QD system in a triplet state as a consequence ${ }^{110}$ of the Pauli exclusion principle. Such a technique effectively allows individual spins to be read by correlating the spin state with the charge state through monitoring the double-QD current 44, 45]. Within this regime, singlet-triplet (S-T) state mixing, relaxation mechanisms, and SO coupling can be probed, since the SO interaction offers a mechanism for coupling hole spin with their orbital motion.

In this work, we investigate the coupling and SO interaction of disorder based states present in p-type Si MOSFETs through PSB, achieved via tuning gate and well voltage to form a double-QD. This allows the determination of the tunnel coupling $\left(t_{c}\right)$ and SO length $\left(l_{S O}\right)$, which offers a direct measure of the SO interaction strength and therefore a method to evaluate the potential of a given system for electricfield mediated control, as well as high frequency spin manipulation. As a result, we take advantage standard Si transistors to find enhanced SO effects of disorder QDs at the oxide interface and obtain a $l_{S O}$ much shorter than anticipated using Si QDs.

\section{Methodology}

(a)

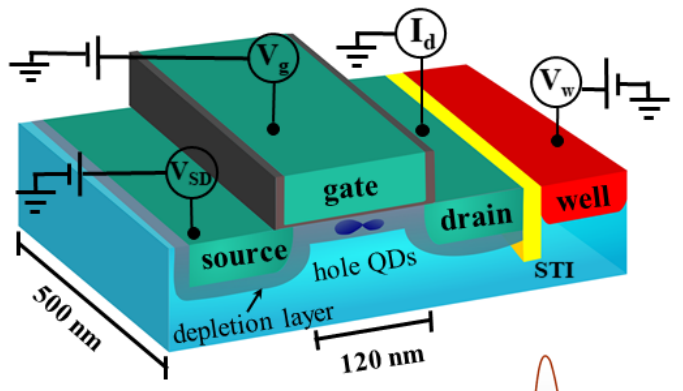

(b)
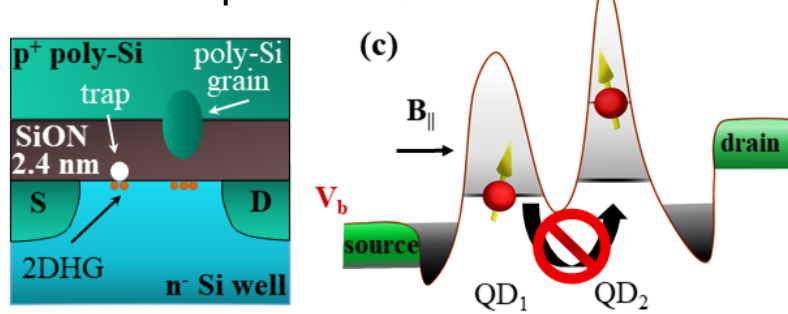

Figure 1. (a) Schematic of p-type Si MOSFET sample with channel dimensions $500 \mathrm{~nm}$ width and $120 \mathrm{~nm}$ length. (b) Crosssection of device displaying disorder at the Si-oxide interface leading to a 2DHG forming below defects. (c) Energy level diagram showing valance band bending which leads to doubleQD formation and the conditions for PSB.

A schematic of the Si p-type MOSFET sample measured is displayed in Figure 1.(a), with a $2.4 \mathrm{~nm}$ thick SiON gate dielectric and a highly doped poly-Si gate with a channel length/width of $120 \mathrm{~nm}$ and 500 $n m$ respectively. As the channel becomes inverted a 2 dimensional hole-gas (2DHG) begins to form and single charging characteristics from QDs can be observed 
due to quantum confinement. In our device, this is a consequence of surface edge roughness by poly-Si grains in the gate and traps at, or close to the Si-oxide interface based on the charging properties observed, as depicted in Figure 1,(b) 46, 47, 48, 49. Typically temperatures below $4 \mathrm{~K}$ are sufficient to observe single hole tunneling due to discrete energy levels in such QDs. All measurements here were carried out within an Oxford instruments ${ }^{4} \mathrm{He}$ cryostat at a temperature of $1.6 \mathrm{~K}$.

Our method to electrically characterize QDs is via $I-V$ characteristics using Yokogawa source meters. This is achieved through biasing the gate $\left(V_{\mathrm{g}}\right)$ and source $\left(V_{\mathrm{SD}}\right)$ terminals while measuring current from a grounded drain terminal $\left(I_{\mathrm{d}}\right)$, to generate a charge stability diagram (CSD). Coulomb diamonds (CDs) jo4 appear as a result of CB (Coulomb blockade) in thers sub-threshold region, whereby transport is blocked6o due to the electrostatic energy and level splitting ${ }_{67}$ of holes occupying the QD, which can be lifted bycs the capacitively coupled gate and source terminalsi69 This not only allows information on the size andro couplings of QDs to be calculated, but also ther1 presence of multiple CDs overlapping highlight doublet72 QD transport features, a key requirement for $\mathrm{PSB}_{73}$ (Figure 1.(c)) in order to study SO effects. We use ar4 p-type MOSFET in particular to take advantage of the 75 enhanced SO interaction of valance bane holes, owing 76 to their p-orbital nature 33 .

\section{Results and Discussion}

The resulting CSDs are displayed in Figure 2, wher®s differential conductance $\left(d I_{\mathrm{d}} / d V_{\mathrm{SD}}\right)$ is plotted to aid82 in highlighting finer features outside $\mathrm{CB}$, includings excited states, and most importantly, to betters visualize PSB. This method is more effective than 8 plotting current, where the magnitude can vary as as6 result of offsets from both triplet leakage and parasiti@87 current paths through the channel. The experimentalss data is plotted in Figure 2.(a)-(c), with simplified8s CSDs drawn in Figure 2.(d)-(f) to better visualize the significant changes. $\mathrm{CB}$ appears as blue in Figureg 2 (a)-(c), with red denoting high $d I_{\mathrm{d}} / d V_{\mathrm{SD}}$ due to thegr availability of levels for single hole transport. Amplifiers current saturation occurred at $1.3 \mathrm{nA}$ producing blues regions at high $V_{\mathrm{g}}$ and $V_{\mathrm{SD}}$. Each $\mathrm{CD}$, corresponding95 to blockaded transport within the QD system, ares marked by a letter and number in Figure 2.(d)-(f). A97 well voltage $\left(V_{\mathrm{w}}\right)$ of $1 \mathrm{~V}$ is applied in Figure 2 (d) $)_{198}$ and a series of largely closed CDs are observed. Thisg9 suggests that transport is occurring through a singleoo QD, where we interpret CDs A1-C1 from QD2, andor $\mathrm{CD}$ D1 from $\mathrm{QD}_{1}$. The energy band diagram (EBDłor depicting this situation is shown in Figure 2, (g) foros
Table 1. Single hole charging properties of CDs as labelled in Figure 2

\begin{tabular}{cccc}
$V_{\mathrm{w}}(V)$ & $\mathrm{CD}(\mathrm{No})$. & $E_{\mathrm{c}}(\mathrm{meV})$ & $C_{g}(\mathrm{aF})$ \\
\hline 1 & $\mathrm{~A} 1$ & 13.3 & 10.4 \\
1 & $\mathrm{~B} 1$ & 6.3 & 24.6 \\
1 & $\mathrm{C} 1$ & 5.5 & 28.6 \\
1 & $\mathrm{D} 1$ & 12.5 & 11.8 \\
2 & $\mathrm{~A} 2$ & 8.9 & 13.3 \\
2 & $\mathrm{~B} 2$ & 16.1 & 9.8 \\
2 & $\mathrm{C} 2$ & 12.7 & 10.4 \\
3 & $\mathrm{~A} 3$ & 10.3 & 11.7 \\
3 & $\mathrm{~B} 3$ & 19.8 & 7.2 \\
3 & $\mathrm{C} 3$ & 12.1 & 11.1
\end{tabular}

the region marked by the star in Figure 2.(d). Due to the small size of the CDs B1 and C1 in Figure 2.(d), single QD transport can occur as a consequence of a reduction in tunneling barrier distance between the source and drain due to the larger QD size, shown schematically in the valence band diagram in 2.(g). When $V_{\mathrm{w}}=2 \mathrm{~V}$ in Figure 2.(b) the CSD changes significantly from the shift and overlap of CDs, in particular we note the appearance of a low $d I_{\mathrm{d}} / d V_{\mathrm{SD}}$ region enclosed by the red dotted line outside of the CD B2 for positive $V_{\mathrm{SD}}$ in Figure 2. (e). This can be interpreted as an initial indicator of PSB, since the transport path will not be completed blockaded due to a slightly elevated $I_{d}$ from triplet leakage that leads to a non-zero, low $d I_{\mathrm{d}} / d V_{\mathrm{SD}}$. We find the optimum contrast is at a threshold of $0.05-0.1 \mathrm{nS}$ to make the PSB region most visible. CB of the dominant transport path also expands considerably between Figure 2. (a)(b), from the enhanced confinement of the smaller CDs observed in Figure 2.(d) as a result of the large scale changes. $\mathrm{QD}_{1}$ emerges from a partially joined potential profile in Figure 2.(g)-(h) which separates due to the application well voltage forming a tunnelling barrier between the QDs as well as increasing the confinement of $\mathrm{QD}_{2}$. The valance band schematic given in Figure 2.(h) during PSB highlights the important changes leading to this result, such as the formation of a weakly coupled double QD through a modified tunneling barrier between them. The number of confined holes in each QD also now satisfies the conditions of PSB, due to the spin selective nature of the second hole entering $\mathrm{QD}_{2}$. Therefore a combination of both the appropriate number of confined holes and the extended region of low $d I_{\mathrm{d}} / d V_{\mathrm{SD}}$ strongly suggests PSB. Upon increasing $V_{\mathrm{w}}$ further to $3 \mathrm{~V}$ in Figure 2. (c) the CSD pattern, and therefore the dominate transport path, largely resembles that of Figure 2. (b) with the exception of the $\mathrm{B} 2$, which displays an extended $\mathrm{CB}$ and the absence of the unique low $d I_{\mathrm{d}} / d V_{\mathrm{SD}}$ feature highlighted previously when comparing Figure 2(e) and (f). This can be 


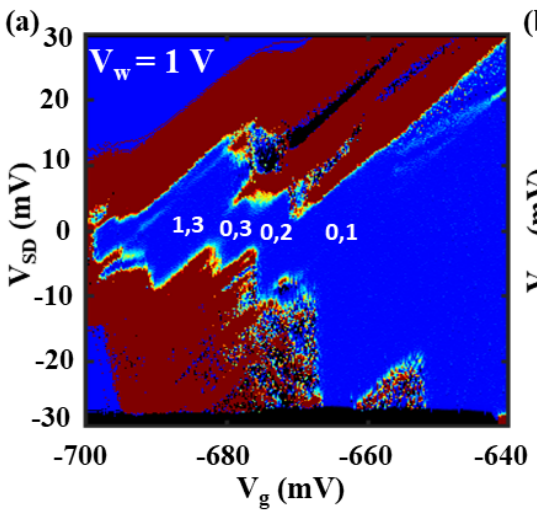

(d)

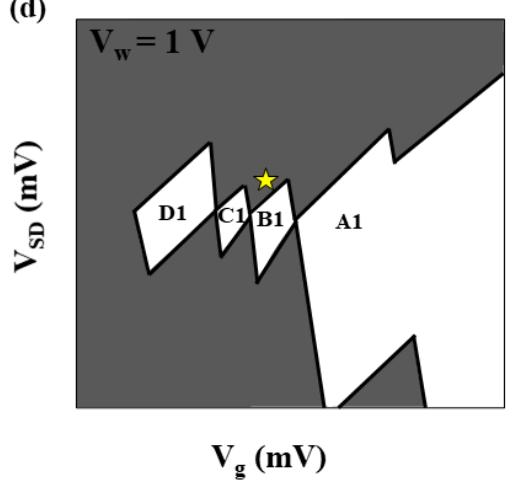

(g) $\hat{W}$

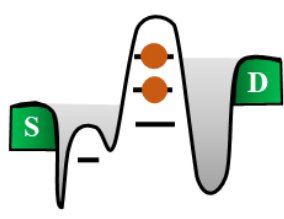

$\mathbf{Q D}_{1} \mathbf{Q D}_{2}$

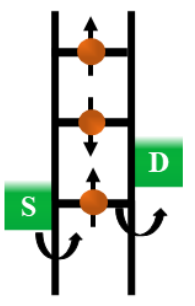

(b)

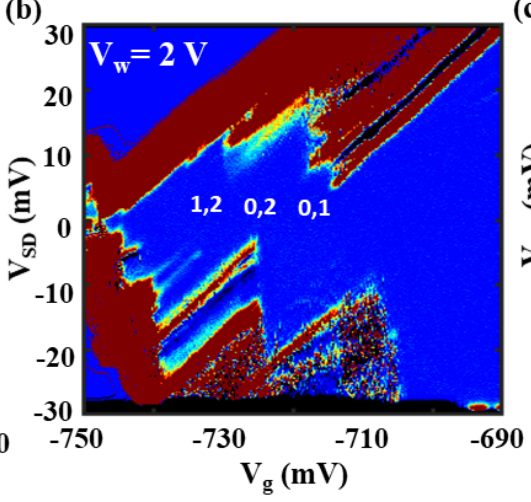

(e)

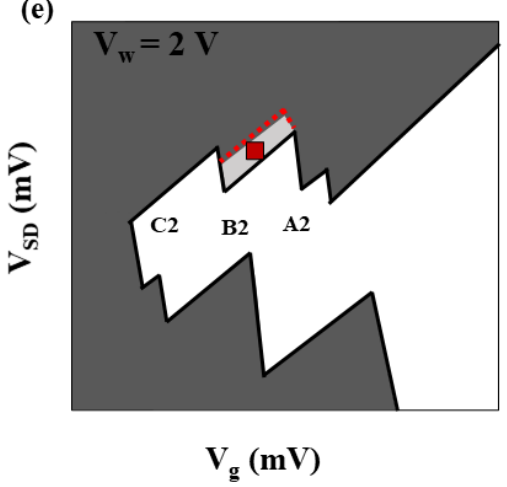

(h)

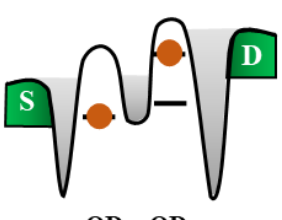

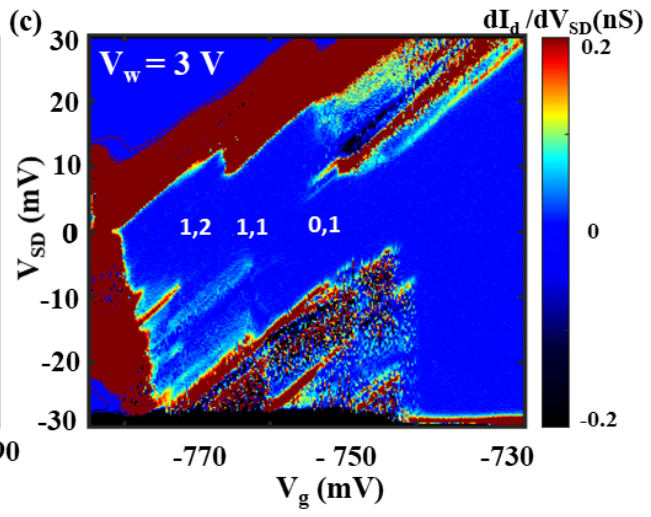

(f)

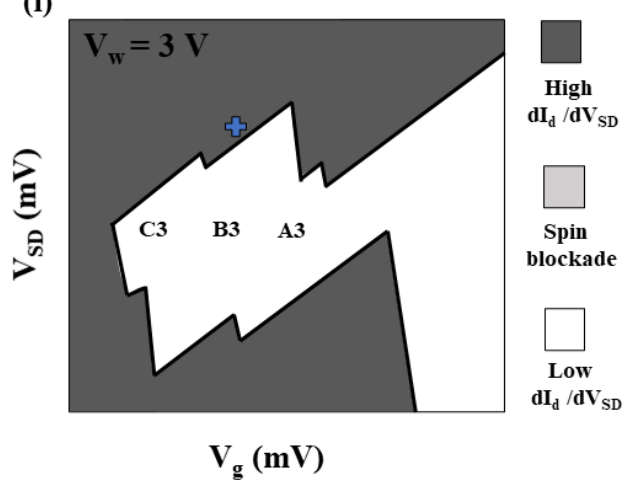

(i) (i) 辽

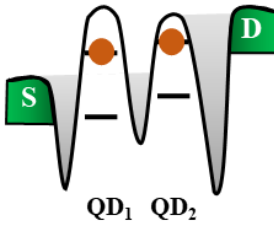

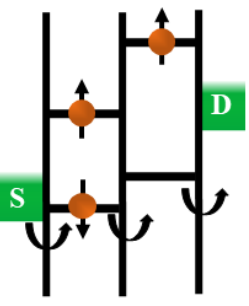

Figure 2. Charge stability diagrams (CSDs) for $V_{\mathrm{SD}}=-30$ to $30 \mathrm{mV}$ over a $60 \mathrm{mV}$ gate voltage $\left(V_{\mathrm{g}}\right)$ range at different well voltages $\left(V_{\mathrm{w}}\right)$. Within the Coulomb diamonds (CDs) the number of confined holes in each $\mathrm{QD}\left(\mathrm{QD}_{1}, \mathrm{QD}_{2}\right)$ are labelled. (a) $V_{\mathrm{w}}=1 \mathrm{~V}$, a row of single CDs appear with various charging energies. (b) $V_{\mathrm{w}}=2 \mathrm{~V}$, the alignment of the QD energy levels change, resulting in overlapping of the CDs which strongly suggests double-QD transport. (c) $V_{\mathrm{w}}=3 \mathrm{~V}$, the alignment of the CDs is further altered, leading to regions of extended Coulomb blockade. The confinement of an additional hole within the dominant transport path is marked by letters A, B, C and D in simplified CSDs (d)-(f), where the number refers to the $V_{\mathrm{w}}$ magnitude, however the same letter does not necessarily denote the same QD energy level in each CSD. The appearance of a PSB like low $d I_{\mathrm{d}} / d V_{\mathrm{SD}}$ region at the edge of B2 for positive $V_{\mathrm{SD}}$ is annotated in (e), where the absence is noted at the edge of B3 in (f) from a change in coupling between the confined levels. (g)-(i) show energy band diagrams marked by the star, square and cross in (d)-(f) respectively. At $V_{\mathrm{w}}=1 \mathrm{~V}$ the transport is assumed to occur largely through a single $\mathrm{QD}$, upon increasing $V_{\mathrm{w}}$ to $2 \mathrm{~V}$ the confinement changes (from larger CDs), together with the QDs becoming weakly coupled and form a double QD exhibiting PSB. When $V_{\mathrm{w}}=3 \mathrm{~V}$ the energy level alignment shifts between the double QD, removing PSB and allowing transport to occur.

explained by the EBD and valance band schematic inıs Figure 2,(i) for the cross in Figure 2,(f). The energyit level alignment shifts, allowing hole transport through 15 the second level in both QDs. Along with changes ton tunneling barriers and QD energy level alignment ato higher $V_{\mathrm{w}}$ values, a considerable threshold shift is alsøı evident. This is explained by an increasing depletionı layer width causing more positively charged ionized ${ }_{20}$ dopants to be exposed, and therefore requires a more negative gate voltage to compensate to achieve the same 2DHG formation.

Upon further analysis, the physical attributes of the dominant transport path are determined through estimating the dimensions of charge stable regions in Figure 2. for positive $V_{\mathrm{SD}}$. This is achieved by calculating gate capacitance $\left(C_{\mathrm{g}}\right)$ and charging energy $\left(E_{\mathrm{c}}\right)$ according to $E_{\mathrm{c}}=\frac{e^{2}}{C_{\Sigma}}$ and $C_{\mathrm{g}}=\frac{e}{\Delta V_{\mathrm{g}}}$ [50], 
(a)
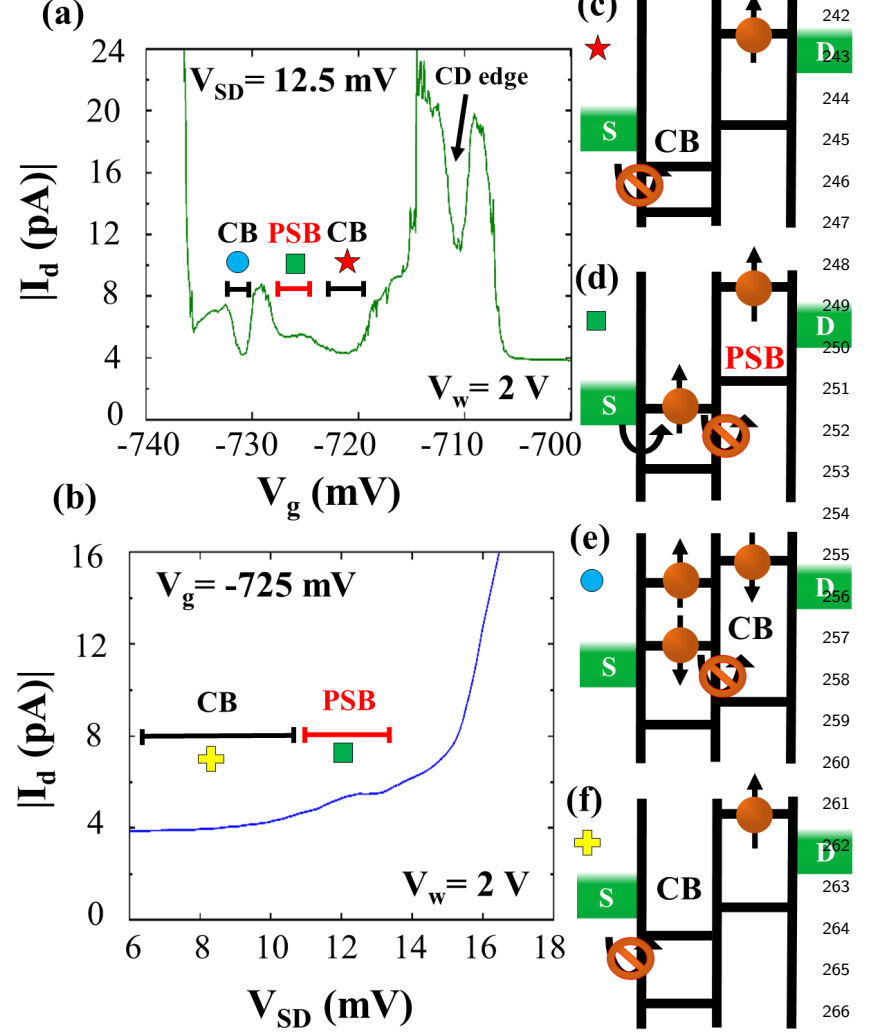

(e)

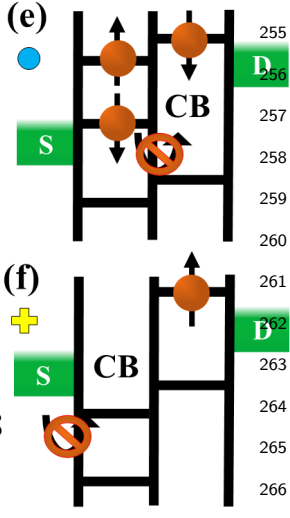

Figure 3. For $V_{\mathrm{w}}$ fixed at $2 \mathrm{~V}$, a gate sweep with $V_{\mathrm{SD}}=$ $12.5 \mathrm{mV}$ is shown in (a), and a source sweep with $V_{\mathrm{g}}=-725_{69}$ $\mathrm{mV}$ is displayed in (b). Coulomb blockade (CB), as well as the suspected Pauli spin blockade (PSB) region from low $d I_{\mathrm{d}} / d V_{\mathrm{SD}}^{270}$ are labelled. (c)-(e) show energy band diagrams marked by the $\mathrm{e}^{71}$ star, square and circle in (a), for the $\mathrm{CB}$ regions as well as $\mathrm{PSB}_{272}$ In (f), an energy band diagram for CB conditions marked by the cross in (b) is displayed, where PSB then occurs at higher $V_{\mathrm{SD}_{274}}$. as the confining potential origin given that fine polySi grain structures reach diameters of the order 50 $\mathrm{nm}$ [52. Although the CSD pattern is similar when comparing Figure 2(b) and (c), the highlighted region in Figure 2 (e) disappeared when $V_{\mathrm{w}}=3 \mathrm{~V}$. As shown schematically in Figure 2 (h), the specific transport path in Figure 2.(b) was no longer available in Figure 2.(c). Such changes which lead to increases in $E_{\mathrm{c}}$ and $C_{\mathrm{g}}$ further demonstrate that the response of each confined level varied according to $V_{\mathrm{w}}$, where the energy level alignment between the QDs shifted, together with the barrier height between them. This is also likely attributable to QD origin, since each QD will have a different capacitive coupling to $V_{\mathrm{w}}$, as with $V_{\mathrm{g}}$, depending on the location and size of the QD as the depletion layer changes 53. The formation of $\mathrm{QD}_{2}$, as the likely product of a poly-Si grain, appears to be more susceptible to $V_{\mathrm{w}}$ from the enlargement of $\mathrm{CD} \mathrm{B} 1$ to $\mathrm{B} 2$ which we ascribe to the same origin, in comparison to the change of $\mathrm{CD} D 1$ to $\mathrm{C} 2$ of $\mathrm{QD}_{1}$ from what is likely an interface trap given the $E_{\mathrm{c}}$.

Focusing on the low $d I_{\mathrm{d}} / d V_{\mathrm{SD}}$ region in Figure 2.(e), a $V_{\mathrm{g}}$ and $V_{\mathrm{SD}}$ sweep at fixed $V_{\mathrm{w}}=2 \mathrm{~V}$ is shown in Figure 3 . (a) and (b) respectively. The $I_{\mathrm{d}}$ valleys in Figure 3 , denote $\mathrm{CB}$, whereas the marginally elevated $I_{\mathrm{d}}$ plateaus correspond to PSB. A dip in current at $V_{\mathrm{g}}$ $=-710 \mathrm{mV}$ signifies the profile sweep nearing a $\mathrm{CD}$ edge, although the system does not enter CB. Figures 3. (c)-(f) show EBDs for the regions labelled by the star, square, circle and cross in Figures 3. (a) and (b). These visually demonstrate how the transport scheme is altered through the application of $V_{\mathrm{g}}$ and $V_{\mathrm{SD}}$. For the star marked in Figure 3.(a), an EBD in Figure 3. (c) shows the system entering CB due to the absence of a level for the first QD. In Figure 3.(d), at larger $V_{\mathrm{g}}$ a level becomes available, but transport is blocked between the QDs due to Pauli selection rules. PSB is temporarily lifted at higher $V_{\mathrm{g}}$ due to the lowering of a level for $\mathrm{QD}_{1}$ within the transport window, however $\mathrm{CB}$ then occurs as displayed in Figure 3. (e) due to a level dropping above the drain for $\mathrm{QD}_{2}$ (circle). An EBD is also given for the CB region preceding PSB at lower $V_{\mathrm{SD}}$ (cross) in Figure 3(f). While an $I_{\mathrm{d}}$ leakage current of $4 \mathrm{pA}$ is observed in the $\mathrm{CB}$ region due to parasitic current paths in the channel, an $I_{\mathrm{d}}$ of $5.6 \mathrm{pA}$ within the PSB region suggests the triplet leakage is 1.6 pA, which is comparable to similar devices exhibiting PSB [41, 40, 31].

Further to the $I_{\mathrm{d}}$ leakage measurements suggesting that the system is in a PSB configuration, much more tangible evidence can be obtained by investigating the magnetic field dependence, offering a window into the prevailing mechanism and any resulting spin related phenomena. Figure 4.(a) shows a higher resolution CSD generated to identify the PSB region clearly. We 

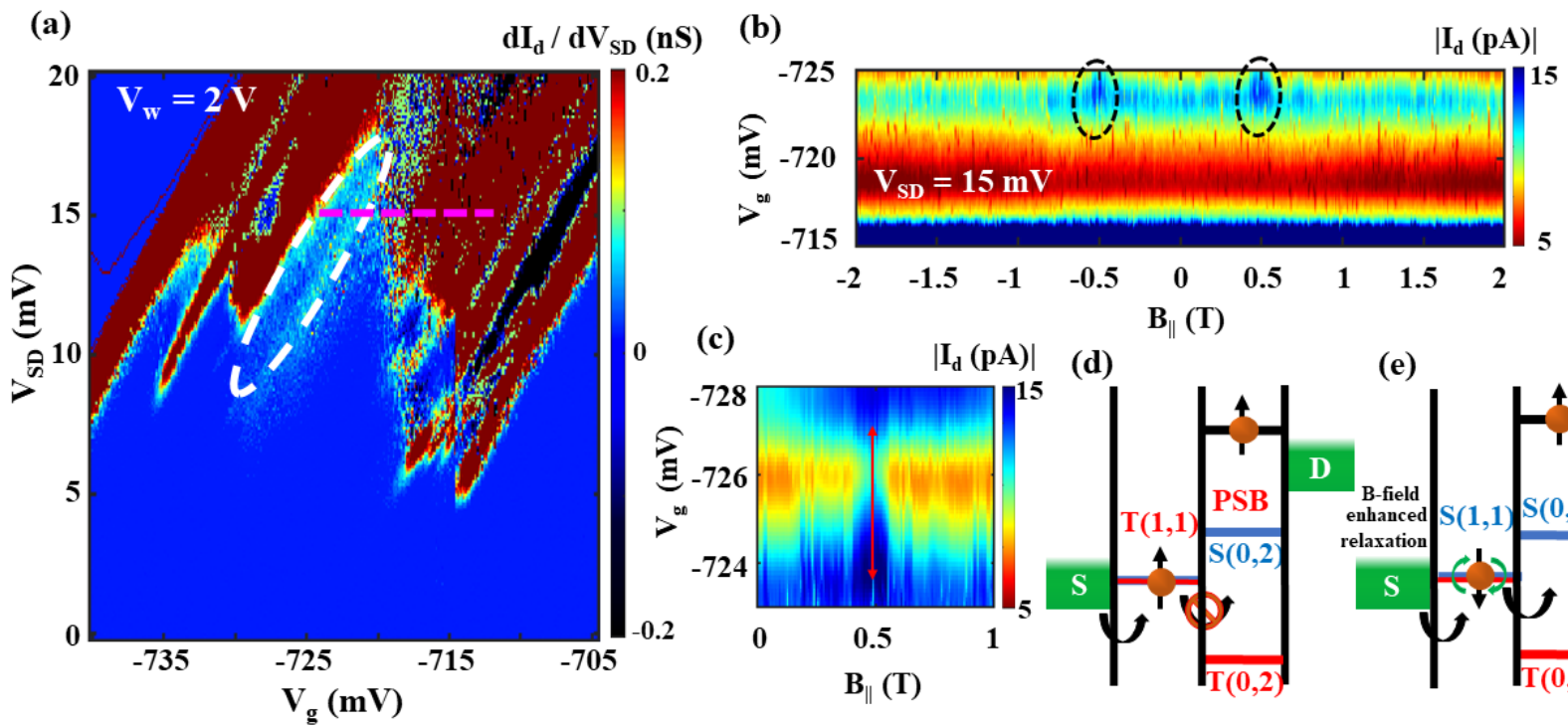

(c)
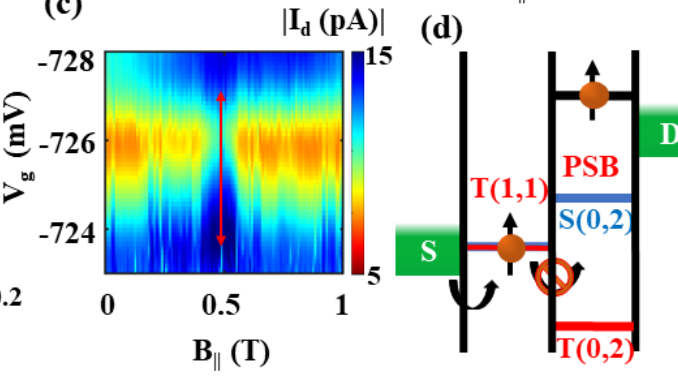

(e)

Figure 4. (a) Charge stability diagram for $V_{\mathrm{SD}}=0$ to $20 \mathrm{mV}$ and $V_{\mathrm{g}}=-705$ to $-740 \mathrm{mV}$ with $V_{\mathrm{w}}=2 \mathrm{~V}$ at the edge of a Coulomb diamond (white), where a reduction in current was observed due to blockaded transport through the two QDs. (b) Current as a function of magnetic field in the PSB region at fixed $V_{\mathrm{SD}}=15 \mathrm{mV}$ between $V_{\mathrm{g}}=-715$ to $-725 \mathrm{mV}$ (pink dashed line in (a)), two peaks are present due to an increase in the PSB leakage current at $0.5 \mathrm{~T}$. (c) High resolution magnetic field spectroscopy scan with a clearly identifiable peak, where the extent of the maximum magnetic field enhanced spin relaxation current with respect to $V_{g}$ is indicated by the red arrow. (d) Energy level diagrams for PSB occurring in a double QD, where the path to $\mathrm{S}(0,2)$ is blocked due to parallel spins in each QD, and (e) where the blockade is partially lifted due to enhanced relaxation from S-T mixing when a finite magnetic field is applied.

focus on the area within the white dashed-circle inzo Figure 4.(a) and apply a magnetic field parallel to27 the channel to investigate spin transport relating tors PSB, where a parallel field is applied to the channed29 to minimize orbital effects 28. Figure 4. (b) shows3o $I_{\mathrm{d}}$ as a function of B-field from $-2 \mathrm{~T}$ to $2 \mathrm{~T}$ for $V_{\mathrm{g}^{31}}$ $=-715 \mathrm{mV}$ to $-725 \mathrm{mV}$ along the pink dashed lin@ 2 in Figure 4. (a). Two peaks become visible at \pm 0.533 $\mathrm{T}$ due to an elevation in $I_{\mathrm{d}}$ from around $10 \mathrm{pA}$ to34 $15 \mathrm{pA}$. A higher resolution B-field sweep from 0 to35 $1 \mathrm{~T}$ is displayed in Figure 4. (c). Here the $I_{\mathrm{d}}$ peaks6 centred around $0.5 \mathrm{~T}$ can be observed to extend over a37 $V_{g}$ region of approximately $-723.5 \mathrm{mV}$ to $-727.5 \mathrm{mV}_{33}$ (marked by red arrow), which closely matches theзs voltage space dimensions of the low $d I_{\mathrm{d}} / d V_{\mathrm{SD}}$ in Figuræ40 4.(a). The resulting magnetic field dependant peaks41 appear due to different relaxation processes competing 42 with each other from a combination of both the $\mathrm{SO}_{43}$ interaction and the Zeeman effect on account of the 44 applied magnetic field, where the latter becomes more 45 efficient at higher fields and therefore allows transition\$46 between S-T configurations 31. Based on this, the ${ }_{47}$ peaks at a finite field are explained by the lifting 48 of PSB as a result of strong spin-orbit induced $\mathrm{S}^{-\mathrm{T}_{49}}$ mixing [32. In Figure 4.(d)-(e) energy level diagramsso are displayed, where the singlet and triplet states are represented by $\mathrm{S}$ and $\mathrm{T}$ respectively, and $\left(N_{\mathrm{QD}_{1}}, N_{\mathrm{QD}_{2}}\right)^{{ }^{51}}$ refers to the number of holes in the highest occupied orbitals of $\mathrm{QD}_{1}$ and $\mathrm{QD}_{2}$. In the absence of a magneti $\varsigma_{52}$ field only the $S(1,1)$ state can provide a direct route to $\mathrm{S}(0,2)$ which are tunnel coupled by $t_{c}$, and PSB occurs if the system enters the $\mathrm{T}(1,1)$ state (Figure 4.(d)). The blocked $\mathrm{T}(1,1)$ states with parallel spins can be lifted however by both relaxation to the $\mathrm{S}(1,1)$ state and $\mathrm{S}(0,2)$ through mixing on account of the SO interaction introducing non spin-conserving tunnelling between the QDs. When a magnetic field is applied the blocked triplet states are split by the Zeeman energy which effectively alters the relaxation rate by providing faster alternative routes between the $\mathrm{T}(1,1)$ and $\mathrm{S}(0,2)$ mixed states and leads to an increase in $I_{d}$ leakage (Figure 4. (e)).

The enhanced relaxation rate is mediated by the SO coupling and proportional to $\alpha B$, where alpha is a ratio characterizing the relative strength of tunnel and spin orbit couplings $t_{c}$ and $t_{S O}$, and $\mathrm{B}$ is the applied field. As such this leads to significant changes when the enhanced leakage becomes comparable to $\Gamma_{r e l}$, the average relaxation rate at zero field from $(1,1)$ to $S(2,0)$, and therefore a noticeable change in leakage $I_{\mathrm{d}}$ due to the lifting of PSB becomes apparent. A model to describe the leakage $I_{\mathrm{d}}$ due to this mechanism as detailed in [30] is given by the equation (1).

$$
\begin{gathered}
I_{\mathrm{d}}(B)=\Gamma_{r e l} \frac{\left[\omega-B^{2}+\tau^{2}\right]\left[\omega(1+4 \gamma)+B^{2}-\tau^{2}\right]}{6 \gamma \omega^{2}+2 \mathrm{~B}^{2} \alpha^{2} \mathrm{t}_{\mathrm{c}}^{2}} \\
\text { Here, } \omega=\sqrt{\left(B^{2}-\tau^{2}\right)^{2}+8 B^{2} \alpha^{2} t_{c}^{2}}, \gamma=\Gamma_{r e l} / \Gamma
\end{gathered}
$$


(where $\Gamma$ is the transfer rate from $\mathrm{S}(0,2)$ to the drain) and $\tau=t_{c} \sqrt{1+3 \alpha^{2}}$ which is the total coupling energy. To convert an applied magnetic field to the energy splitting; the Zeeman energy is calculated via $\Delta E_{\mathrm{z}}=$ $g \mu_{\mathrm{b}} B_{\|}$, where $\mu_{\mathrm{b}}$ is the Bohr magnetron and $g$ is the gyro-magnetic ratio which we assume to be $\approx 2$. We apply this theory in the form of fitting (red) to the data in Figure 5, where a single point centered around the site of PSB at $V_{\mathrm{g}}=-724 \mathrm{mV}$ is plotted. A zero field dip can be noted, together with two clear leakage $I_{\mathrm{d}}$ peaks at $\pm 0.5 \mathrm{~T}$ with a magnitude of around 5 pA. As discussed earlier a parasitic current path elsewhere through the channel led to an additional current measured within $\mathrm{CB}$, therefore we subtracted this offset, as well as the current from an isolated charge trap event, to produce a PSB leakage current of around $1.6 \mathrm{pA}$ at zero field. With the combination of the known splitting energy and equation (1), we extract a $t_{c}$ of $57 \mu \mathrm{eV}$ from the data, which is in good agreement for gate defined silicon QDs in a MOSFET device with similar dimensions 10. Since $\alpha$ is a ratio that can be used to parametize the QD diameter to $l_{S O_{4}^{408}}$ it enables a way to gauge the SO interaction strengthos relative to the confining potential, where $l_{S O}$ describesio the ballistic distance travelled by a hole relative to it ${ }^{11}$ spin precession [41, 54. Due to this, $l_{S O}$ is related t ${ }^{12}$ $d_{Q D}$ via $l_{S O} \approx\left(t_{c} / t_{S O}\right) d_{Q D}$, which for a calculated ${ }^{13}$ $d_{Q D}$ of around $30 \mathrm{~nm}$ using Figure 2. (e) gives an $l_{S \mathcal{O}^{14}}$ value of $250 \mathrm{~nm}$.

Comparing the $l_{S O}$ obtained with others reported ${ }^{16}$ in $\mathrm{Si}$ reveals that our value is almost two orders of ir magnitude smaller than bulk $\mathrm{Si}$, where $20 \mu \mathrm{m}$ has been measured [55]. We also obtain a much lower ${ }_{18}$ value when compared to a $\mathrm{Si}$ spin qubit device with an $l_{S O}$ of $1 \mu \mathrm{m}$ which was achieved using electron based19 QDs. Lengths as low as $110 \mathrm{~nm}$ have been estimated20 in Si but using heavy holes in a planar multi-gate21 defined QD device, operating at temperatures an order22 of magnitude smaller with reference to our MOSFET $\mathrm{M}_{23}$ device [34, 32. Generally speaking our results indicate24 that the $l_{S O}$ of Si disorder QDs characterized here are25 closer to those measured in III-IV materials which arezo typically $\approx 130-250 \mathrm{~nm} 29$, 56, 41. It should be noted27 that single hole transport here is only possible owing tore QD formation in such devices as a result of defects atze the interface. Whether they are local to the gate, suchas as poly-Si grains and traps, or other impurities which 31 create variable potential profile, are largely a naturał 32 consequence of stochastic processes. Although thæ3 inability to control the number of QDs in this type of 34 system remains, the use of the well allowed control over35 the transport properties within a multi-level systenas6 which lead to the emergence of PSB. The creation of 37 such a configuration yielded a relatively strong $\mathrm{SO}_{38}$ interaction given the $l_{S O}$ extracted. Such an effects9

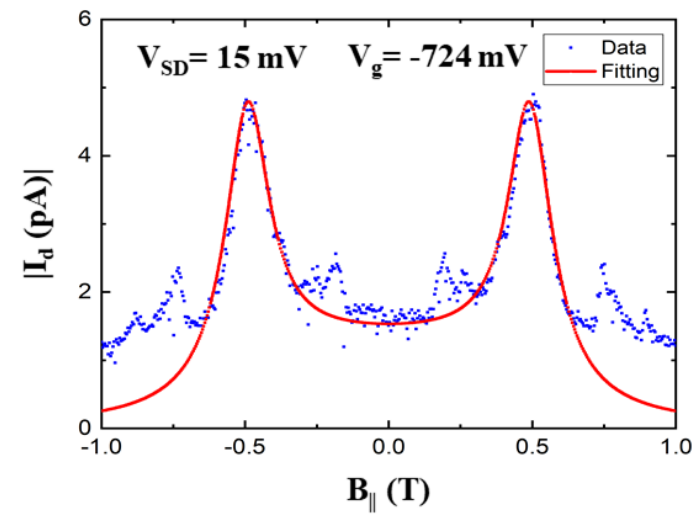

Figure 5. A profile plot showing the magnetic field dependant leakage current (blue) of a single point centered within the PSB region at a $V_{\mathrm{SD}}$ of $15 \mathrm{mV}$ and $V_{\mathrm{g}}=-724 \mathrm{mV}$. Peaks begin to appear at $\pm 0.5 \mathrm{~T}$ where a leakage current of around $5 \mathrm{pA}$ is observed. Using equation (1) a fitting is applied to the peaks (red) to extract a $t_{c}$ of $57 \mu \mathrm{eV}$ and an $l_{S O}$ of $250 \mathrm{~nm}$.

has been previously attributed to inversion asymmetry at the interface possibly due to position dependent electric fields at the oxide boundary [34. Therefore our results support engineering strong SO interactions at the interface in $\mathrm{Si}$, as such we envisage more focus on exploring the useful properties of disorder QDs, particularly at the Si-oxide interface. This may well be of interest for industries where standard MOSFETs can act as a testing platform for quantum information processing schemes.

\section{Conclusion}

To conclude, pseudo control over the QD confinement potentials originating at the Si-oxide interface can be achieved as a consequence of well tuning to shift the energy level alignment and coupling between two QDs, allowing double QD transport phenomena to be probed in this type of system. This enabled spin related transport properties to be investigated through PSB, producing a significantly short $l_{S O}$ of $250 \mathrm{~nm}$ as well as a $t_{\mathrm{c}}$ of $57 \mu \mathrm{eV}$. Our work therefore highlights a path for exploring alternative quantum information technologies using disorder based QDs by accommodating their advantageous $\mathrm{SO}$ properties at the interface on an accessible platform such as industry standard Si MOSFETs.

This work is supported by EPSRC Manufacturing Fellowship (EP/M008975/1) and Lloyds Register Foundation International Consortium of Nanotechnology.

The data that support the findings of this study are openly available in Southampton ePrint research repository at: https://doi.org/10.5258/SOTON/D1485 


\section{References}

[1] Ladd T D, Jelezko F, Laflamme R, Nakamura Y, Monroẹ13 C and O'Brien J L 2010 Nature 46445

[2] Veldhorst M, Eenink H G J, Yang C H and Dzurak A S15 2017 Nat. Commun. 81766

[3] Huang W, Yang C H, Chan K W, T Tanttu B H, Leoki17 R C C, Fogarty M A, J C C Hwang F E H, Itoh K M5,18 Morello A, Laucht A and Dzurak A S 2019 Nature 56919 532

[4] Ferraro E and Prati E 2020 Phys. Lett. A. 384126352

[5] Loss D and DiVincenzo D P 1998 Phys. Rev. A. 57120522

[6] Takahashi Y, Ono Y, Fujiwara A and Inokawa H $2002 \quad 5.23$ Phys. Condens. Matter 14 R995

[7] Hanson R, Kouwenhoven L P, Petta J R, Tarucha S and25 Vandersypen L M K 2007 Rev. Mod. Phys. $791217 \quad 526$

[8] Stegner A R, Boehme C, Huebl H, Stutzmann M, Lips I₹27 and Brandt M S 2006 Nature Phys. 2835

[9] Morello A, Pla J J, Zwanenburg A, Chan K W, Tan K Y, 529 Huebl H, Mottonen M, Nugroho C D, Yang C, variso Donkelaar J A, Alves A D C, Jamieson D N, Escot蛙 C C, Hollenberg L C L, Clark R G and Dzurak A \$\$\$32 2010 Nature 467687

[10] Eenink H G J, Petit L, Lawrie W I L, Clarke J S an\& ${ }^{34}$ Vandersypen L M K 2019 Nano. Lett. 198653

[11] Xiao M, House M G and Jiang H W 2010 Phys. Rev. Let5.36 104096801

[12] Yang C H, Rossi A, Ruskov R, Lai N S, Mohiyaddin F A, 538 Lee S, Tahan C, Klimeck G, Morello A and Dzurak A \$\$39 2013 Nat. Commun. 42069

[13] Maurand R, Jehl X, Kotekar-Patil D, Corna A, Bo541 huslavskyi H, ville R L, Hutin L, Barraud S, Vinet M542 Sanquer M and Franceschi S D 2016 Nat. Commun. ₹ $^{43}$ 13575

[14] URL https://www.imec-int.com/en/articles/moving ${ }^{5 / 5}$ quantum-computing-lab-fab

[15] Petta J R, Johnson A C, Taylor J M, Laird E A, Yacoby47 A, Lukin M D, Marcus C M, Hanson M P and Gossark48 A C 2005 Science 3092180

[16] Zwanenburg F A, Dzurak A S, Morello A, Simmons M Y5,50 Hollenberg L C L, Klimeck G, Rogge S, Coppersmith S N\$51 and Eriksson M A 2013 Rev. Mod. Phys. 85961

[17] Shor P W 1995 Phys. Rev. A. 522493

[18] Pla J J, K, Tan Y, Dehollain J P, Lim W H, Morton J J L554 F A Zwanenburg D N J, Dzurak A S and Morello A 201855 Nature 496334

[19] Kawakami E, Scarlino P, Ward D R, Braakman F R, Savage57 D E, Lagally M G, Friesen M, Coppersmith S N, Erikssok ${ }^{58}$ M A and Vandersypen L M K 2014 Nat. Nanotechnol. 959 666

[20] Pla J J, Tan K Y, Dehollain J P, Lim W H, Morton J J L5, Jamieson D N, Dzurak A S and Morello A 2012 Naturéc 489541

[21] Terhal B M 2015 Rev. Mod. Phys. 87307

[22] Xiao M, Martin I and Jiang H W 2003 Phys. Rev. Lett. 9165 078301

[23] Xiao M, Martin I, Yablonovitch E and Jiang H W 200467 Nature 430435

[24] Li J, Pud S, Petrychuk M, Offenhäusser A and Vitusevick69 S 2014 Nano. Lett. 143504

[25] Tanamoto T, Nishi Y and Ono K 2019 Appl. Phys. Let5.71 115033504

[26] Yamahata G, Nishiguchi K and Fujiwara A 2013 Na5.73 Commun 55038

[27] Yamahata G, Giblin S P, Kataoka M, Karasawa T and ${ }^{75}$ Fujiwara A 2017 Sci. Rep. 745137
[28] Ono K, Giavaras G, Tanamoto T, Ohguro T, Hu X and Nori F 2017 Phys. Rev. Lett. 119156802

[29] Fasth C, Fuhrer A, Samuelson L, Golovach V N and Loss D 2007 Phys. Rev. Lett. 98266801

[30] Zarassi A, Su Z, Danon J, Schwenderling J, Hocevar M, Nguyen B M, Yoo J, Dayeh S A, and Frolov S M 2017 Phys. Rev. B. 95155416

[31] Xu G, Gao F, Wang K, Zhang T, Liu H, Cao G, Wang T, Zhang J, Jiang H, Li H and Guo G 2020 App. Phys. Exp. 13

[32] Li R, Hudson F E, Dzurak A S and Hamilton A R 2015 Nano. lett. 157314

[33] Shankar S, Tyryshkin A M, He J and Lyon S A 2010 Phys. Rev. B. 82195323

[34] Harvey-Collard P, Jacobson N T, Bureau-Oxton C, Jock R M, Srinivasa V, Mounce A M, D R Ward J M A, Manginell R P, Wendt J R, Pluym T, Lilly M P, Luhman D R, Pioro-Ladri'ere M and Carroll M S 2019 Phys. Rev. Lett. 122217702

[35] Corna A, L Bourdet a R M, Crippa A, Kotekar-Patil D, Bohuslavskyi H, RLaviéville, Hutin L, Barraud S, Jehl X, Vinet M, Franceschi S, Niquet Y and Sanquer M 2018 npj Quantum Inf. 46

[36] Jock R M, Jacobson N T, Harvey-Collard P, Mounce A M, Srinivasa V, D R Ward J M A, Manginell R P, Wendt J R, Rudolph M, Pluym T, Gamble J K, Baczewski A D, WItszel W M and Carroll M S 2018 Nat. Commun. 9 1768

[37] Ono K, Austing D G, Tokura Y and Tarucha S 2002 Science 2971313

[38] Weber B, Tan Y H M Mahapatra S, Watson T F, Ryu H, Rahman R, Hollenberg L C L, Klimeck G and Simmons M Y 2014 Nat. Nano. 9430

[39] Amasha S, MacLean K, Radu I P, Zumbuhl D M, Kastner M A, Hanson M P and Gossard A C 2008 Phys. Rev. Lett. 100046803

[40] Danon J and Nazarov Y V 2009 Phys. Rev. B. 80041301

[41] Nadj-Perge S, Frolov S M, van Tilburg J W W, Danon J, Nazarov Y V, Algra R, Bakkers E P A M and Kouwenhoven L P 2010 Phys. Rev. B 81201305

[42] Yamahata G, Kodera T, Churchill H O H, Uchida K, Marcus C M and Oda S 2012 Phys. Rev. B. 86115322

[43] Yamaoka Y, Iwasaki K, Oda S and Kodera T 2017 Jpn. J. Appl. 56 04CK07

[44] Liu H W, Fujisawa T, Ono Y, Inokawa H, Fujiwara A, Takashina K and Hirayama Y 2008 Phys. Rev. B. 77 073310

[45] Lai N S, Lim W H, Yang C H, Zwanenburg F A, Coish W A, Qassemi F, Morello A and Dzurak A S 2011 Sci. Rep. 1110

[46] Saito S, Torii K, Shimamoto Y, Tsujikawa S, Hamamura $\mathrm{H}$, Tonomura $\mathrm{O}$, Mine T, Hisamoto D, Onai T, Yugami J, Hiratani M and Kimura S 2004 Appl. Phys. Lett. 84 1395

[47] Li Z, Husain M K, Yoshimoto H, Tani K, Y Sasago D Hisamoto J D F, Kataoka M, Tsuchiya Y and Saito S 2017 Semicond. Sci. Technol. 32075001

[48] Li Z, Sotto M, Liu F, Husain M K, Yoshimoto H, Y Sasago D H, Tomita I, Tsuchiya Y and Saito S 2018 Nat. Sci. Rep. 8250

[49] Ibukuro K, Hillier J W, Liu F, Husain M K, Li Z, Tomita I, Tsuchiya Y, Rutt H N and Saito S 2020 AIP Adv. 10 055025

[50] Grabert H and Devoret M H 1992 Single Charge Tunneling (Plenum Press)

[51] Spruijtenburg P C, Amitonov S V, Mueller F, van der Wiel W G and Zwanenburg F A 2016 Sci. Rep. 638127

[52] Yamauchi N Hajjar J and Reif R 1991 IEEE Trans. Electron Dev. 38 55-60

[53] Ono K, Tanamoto T and Ohguro T 2013 App. Phys. Lett. 
103183107

[54] Zumbuhl D M 2004 Coherence and Spin in GaAs Quantum Dots Ph.D. thesis Harvard, Cambridge

[55] Mehl S and DiVincenzo D P 2014 Phys. Rev. B 90195424

[56] Nadj-Perge S, Pribiag V S, van den Berg J W G, Zuo K, Plissard S R, Bakkers E P A M, Frolov S M and Kouwenhoven L P 2012 Phys. Rev. Lett. 108166801 